\title{
Impact of the spatial laser distribution on photocathode gun operation
}

\author{
Feng Zhou, ${ }^{*}$ Axel Brachmann, Paul Emma, Sasha Gilevich, and Zhirong Huang \\ SLAC National Accelerator Laboratory, 2575 Sand Hill Road, Menlo Park, California 94025, USA
}

(Received 1 May 2012; published 10 September 2012)

\begin{abstract}
It is widely believed that a drive laser with uniform temporal and spatial laser profiles is required to generate the lowest emittance beam at the photoinjector. However, for a given 3 ps smooth-Gaussian laser temporal profile, our recent simulations indicate that a truncated-Gaussian laser spatial profile produces an electron beam with smaller emittance. The simulation results are qualitatively confirmed by later analytical calculation, and also confirmed by measurements: emittance reduction of $\sim 25 \%$ was observed at the linac coherent light source (LCLS) injector with a truncated-Gaussian laser spatial profile at the nominal operating bunch charge of $150 \mathrm{pC}$. There was a significant secondary benefit-laser transmission through the iris for the truncated-Gaussian profile was about twice that compared to the nearly uniform distribution, which significantly loosens the laser power and quantum efficiency requirements for drive laser system and photocathode. Since February 9, 2012, the drive laser with the truncated-Gaussian spatial distribution has been used for LCLS routine user operations and the corresponding free electron laser power is at least the same as the one when using the nearly uniform spatial profile.
\end{abstract}

PACS numbers: 07.77.Ka

\section{OVERVIEW}

It is well known that the cost and performance of the $\mathrm{x}$-ray free-electron-laser (FEL) [1-3] depend critically on the emittance of the electron beam. Modern linear accelerators, such as the Linac Coherent Light Source (LCLS) [1], efficiently preserve the electron beam emittance through acceleration. Consequently, it becomes important to extract electrons from a photoinjector with the lowest possible emittance. Extensive worldwide photoinjectorrelated R\&D aimed at emittance reduction has been performed for more than two decades [4-11]. Many studies suggest that the drive laser pulse must be uniform in time and space at the photocathode to produce the best emittance beam [12,13], and this was the approach first pursued at the LCLS. The LCLS commissioning and early operations started with 3 ps pulses stacked to form 6.5 ps nearly flattop pulses [14] using a split and delay arrangement, which adds complexity to the laser system. A nearly uniform laser spatial profile was obtained by overfilling an iris located far upstream of the cathode. Most of the laser beam was lost on the iris. As such, a very high laser power from the laser amplifier was required to keep sufficient laser energy at the cathode. The laser beam size on the iris can be adjusted by tuning the telescope upstream of the iris.

The LCLS team is constantly making efforts to improve the injector emittance as well as to simplify the drive laser system in order to increase system availability. In the

\footnotetext{
*zhoufeng@slac.stanford.edu
}

Published by the American Physical Society under the terms of the Creative Commons Attribution 3.0 License. Further distribution of this work must maintain attribution to the author(s) and the published article's title, journal citation, and DOI. spring of 2010, one of the stacked lasers pulses was removed, resulting in no obvious emittance change [15]. Since then, 3 ps single polarization with Gaussian temporal profile is used for the user operations. In late 2010, simulations [16] indicated that the emittance could be improved using a truncated-Gaussian laser spatial distribution rather than the uniform distribution regularly used for operations. Generating a truncated-Gaussian beam at the cathode is easy and requires a relatively small laser beam at the iris, with most of the laser beam passing through the iris. Measurements at the LCLS injector using a truncatedGaussian laser spatial profile confirmed the simulation results, namely the emittance was reduced by $\sim 25 \%$.

To summarize, the advantages of using a truncatedGaussian laser spatial profile over a uniform one at the cathode are as follows: (1) lower emittance and (2) larger laser transmission through the iris, thereby improving overall laser system and cathode reliability. This paper will present simulations with truncated-Gaussian laser spatial profiles using the multiparticle tracking code (IMPACTT [17]), theoretical analyses, and experimental results performed at the LCLS injector.

\section{SIMULATIONS AND THEORY}

A full three-dimensional code, IMPACTT, was used to track particles. The code takes into account space charge forces, short range wakefields. All simulation parameters were the same as those routinely used to operate the LCLS injector: 3 ps Gaussian laser pulse (FWHM), peak accelerating gradient at the cathode of $115 \mathrm{MV} / \mathrm{m}$, a laser launch phase of $30^{\circ}$ from zero crossing, $150 \mathrm{pC}$ of bunch charge, and $135 \mathrm{MeV}$ of beam energy. The measured photocathode thermal emittance of $0.9 \mu \mathrm{m} / \mathrm{mm} \mathrm{rms}$ [18] 

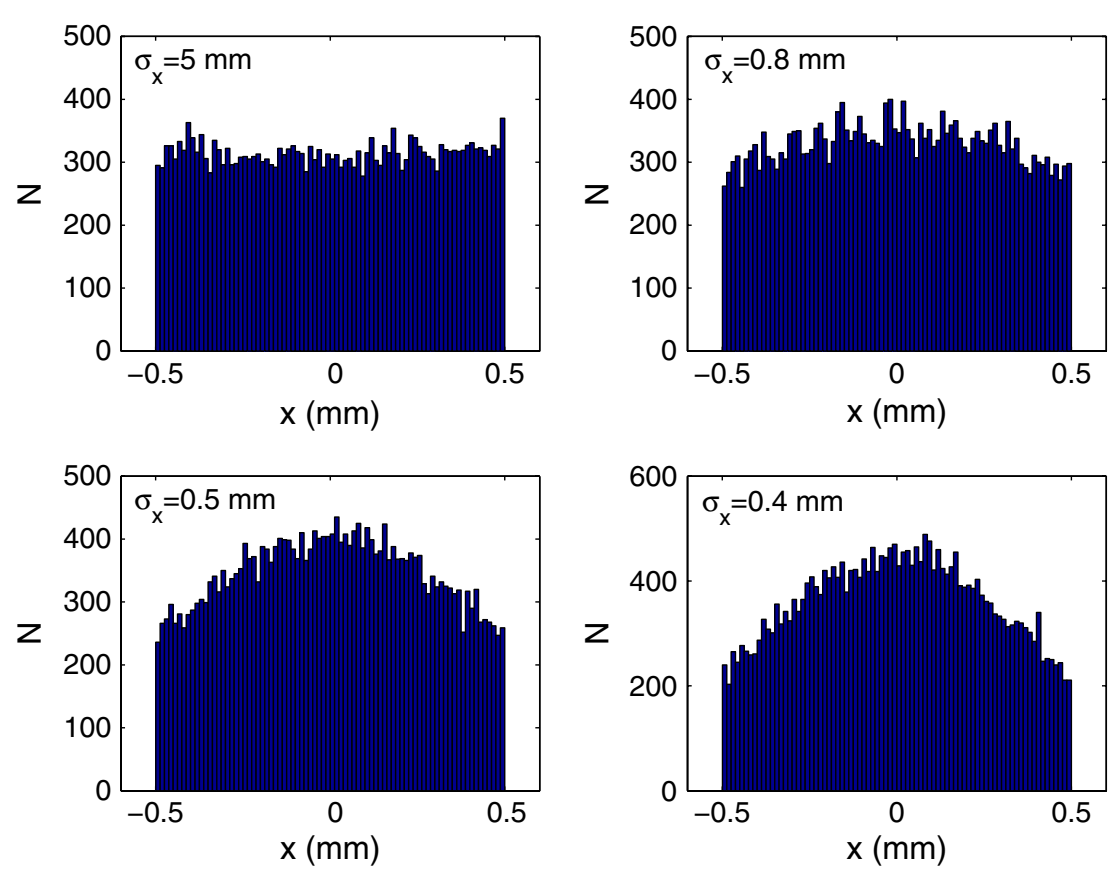

FIG. 1. Lineout intensity of the initial laser spatial distributions used in the simulations: the pure uniform laser spatial beam $\left(\sigma_{x}=5.0 \mathrm{~mm}\right)$, nearly uniform $\left(\sigma_{x}=0.8 \mathrm{~mm}\right)$, and different truncated-Gaussian laser spatial distributions $\left(\sigma_{x}=0.4-0.5 \mathrm{~mm}\right)$.

was included in all simulations. The simulation results for the uniform and truncated-Gaussian laser spatial distributions are compared.

\section{A. Simulated emittance}

The spatial distributions for particles tracking were externally generated using smooth Gaussian profiles but with different standard deviation values $\left(\sigma_{x}\right)$. For LCLS routine operations at a $150 \mathrm{pC}$ bunch charge, optimization of emittance and charge production led to a $1.0 \mathrm{~mm}$ edge to edge laser spot diameter at the cathode. All spatial laser distributions for the simulations are therefore truncated at a radius of $\pm 0.5 \mathrm{~mm}$. To obtain a uniform laser spatial distribution, the $\sigma_{x}$ value of a Gaussian distribution must be much larger than the iris radius $(0.5 \mathrm{~mm}), 5.0 \mathrm{~mm}$ for instance. However, the $\sigma_{x}$ values of the truncated-Gaussian distributions are comparable to or smaller than the iris radius, for example, from 0.3 to $0.5 \mathrm{~mm}$. Figure 1 shows the lineout intensity of different laser spatial profiles in the $x$ plane (same process for $y$ plane) used for the particle tracking. The distribution labeled with $\sigma_{x}=5.0 \mathrm{~mm}$ represents a pure uniform spatial profile, while the ones with $\sigma_{x}=0.4-0.5$ correspond to different truncated-Gaussian spatial profiles. The one with $\sigma_{x}=0.8 \mathrm{~mm}$ is in between the pure uniform and truncated Gaussian, termed nearly uniform. The simulated projected and time-sliced emittances at $135 \mathrm{MeV}$ of beam energy with $150 \mathrm{pC}$ are shown in Fig. 2. Note that $5 \%$ of particles in the tails are excluded in the emittance calculations. It shows that the truncatedGaussian distribution with $\sigma_{x}=0.4 \mathrm{~mm}$ promises the best projected and time-sliced emittances. It also shows the emittance with the pure uniform laser spatial is worse than the one with the nearly uniform profile. The result may explain why the measured LCLS injector emittance was always better than our previous simulations with the LCLS nominal charges, $150-250 \mathrm{pC}$. We had always
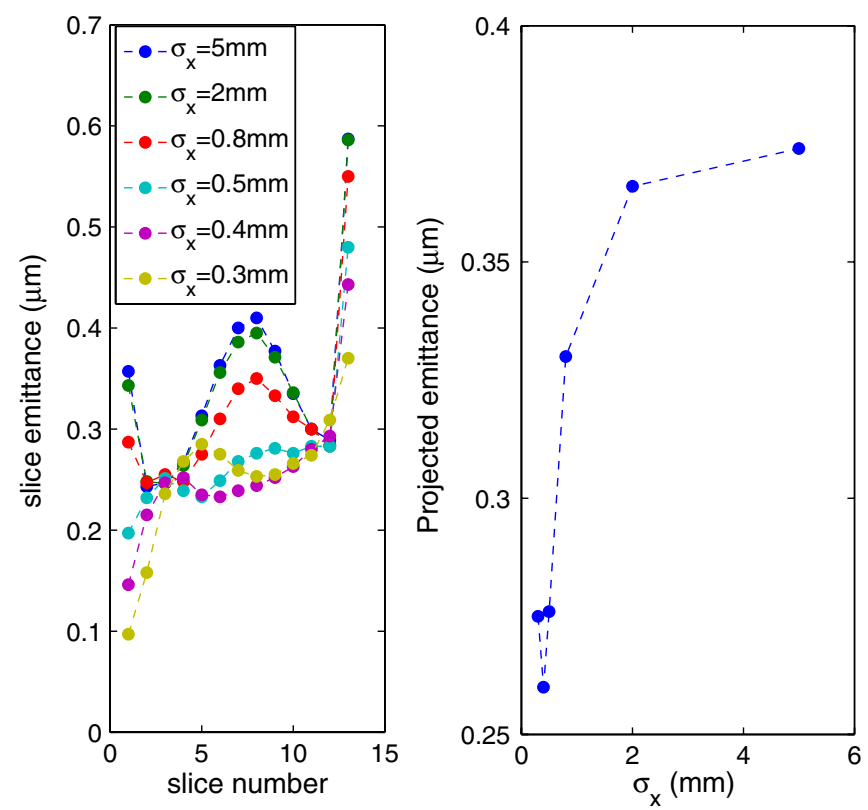

FIG. 2. Projected (left) and time-sliced (right) emittances with the pure uniform $\left(\sigma_{x}=5.0 \mathrm{~mm}\right.$ and $\left.2.0 \mathrm{~mm}\right)$, nearly uniform $\left(\sigma_{x}=0.8 \mathrm{~mm}\right)$, and different truncated-Gaussian beams $\left(\sigma_{x}=\right.$ $0.5,0.4$, and $0.3 \mathrm{~mm}$ ) for a $150 \mathrm{pC}$ bunch charge. Note that $5 \%$ particles in the tails are excluded in the emittance calculations. 
believed that the uniform laser spatial distribution produced the best emittance beam, so a pure uniform laser spatial beam was assumed in the simulations. If the pure uniform laser spatial is replaced with the nearly uniform profile ( $\sigma_{x}=0.8 \mathrm{~mm}$ in Fig. 1 for example), the simulated emittance agrees very well with the measured one, about $0.35 \mu \mathrm{m}$. The rms sizes of the distributions labeled as $\sigma_{x}=5,0.8,0.5$, and $0.4 \mathrm{~mm}$ in Fig. 1 are very close, corresponding to $0.25,0.246,0.24$, and $0.235 \mathrm{~mm}$, respectively. Therefore, the thermal emittance dependence of the distributions is negligible. In other words, the thermal emittance contributions for these different truncated Gaussians are close to each other. The conclusion from the simulations for $150 \mathrm{pC}$ is also valid for $250 \mathrm{pC}$.

\section{B. Analytical model}

A simple analytical model was developed to explain the simulations. We consider the distributions in $x, y$, and $z$ planes as Gaussians for all spatial distributions. The charge density $\rho$ for the truncated-Gaussian profile is

$$
\rho=\frac{Q}{(2 \pi)^{3 / 2} \sigma_{x}^{2} \operatorname{Erf}\left[r /\left(\sqrt{2} \sigma_{x}\right)\right]^{2} \sigma_{z}} e^{-\left[\left(x^{2}+y^{2}\right) / 2 \sigma_{x}^{2}\right]-\left(z^{2} / 2 \sigma_{z}^{2}\right)},
$$

where $Q$ is the total charge, Erf is an error function, $r$ is the beam radius, $\sigma_{x}$ and $\sigma_{z}$ are the rms beam sizes in transverse and longitudinal planes, and $\rho=0$ for $|x|>r$ or $|y|>r$ due to the transverse truncation (approximated by a square iris). The solution of the electrostatic potential $\phi$ is

$\phi(x, y, z)=\frac{1}{4 \pi \varepsilon_{0}} \int \frac{\rho\left(x^{\prime}, y^{\prime}, z^{\prime}\right) d x^{\prime} d y^{\prime} d z^{\prime}}{\left[\left(x-x^{\prime}\right)^{2}+\left(y-y^{\prime}\right)^{2}+\left(z-z^{\prime}\right)^{2}\right]^{1 / 2}}$.

Following Eqs. (53) and (54) from Ref. [19], the transverse space charge field derived from the above electrostatic potential can be expressed as a one-dimensional integral. The bunch is pancake-like with a large aspect ratio $x / z$ when it is immediately released from the cathode. For the numerical illustration shown here, we take $\sigma_{z} / r=$ 0.1 . The transverse space charge force $E_{x}$ near the cathode is calculated for various $\sigma_{x} / r$ as shown in Fig. 3. The $x$ (and $y$ ) range of interest used for the numerical integration of our problems extends from $-r$ to $r$, where $r$ is the iris radius (i.e. $0.5 \mathrm{~mm}$ ). All spatial distributions are truncated at $\pm 0.5 \mathrm{~mm}$, consistent with the simulations. In Fig. 3, the black curve with $\sigma_{x} / r=10$ (i.e., $\sigma_{x}=5.0 \mathrm{~mm}$ ) is for the uniform spatial beam while the red curve is for the truncated Gaussian with $\sigma_{x}=0.5 \mathrm{~mm}$. The blue one is for truncated Gaussian with $\sigma_{x}=0.3 \mathrm{~mm}$. It is clear that the transverse space charge force of the truncated-Gaussian distribution with $\sigma_{x}=\sim 0.5 \mathrm{~mm}$ is the most linear result, which may produce a better transverse emittance. This analysis, although highly simplified and assuming a square

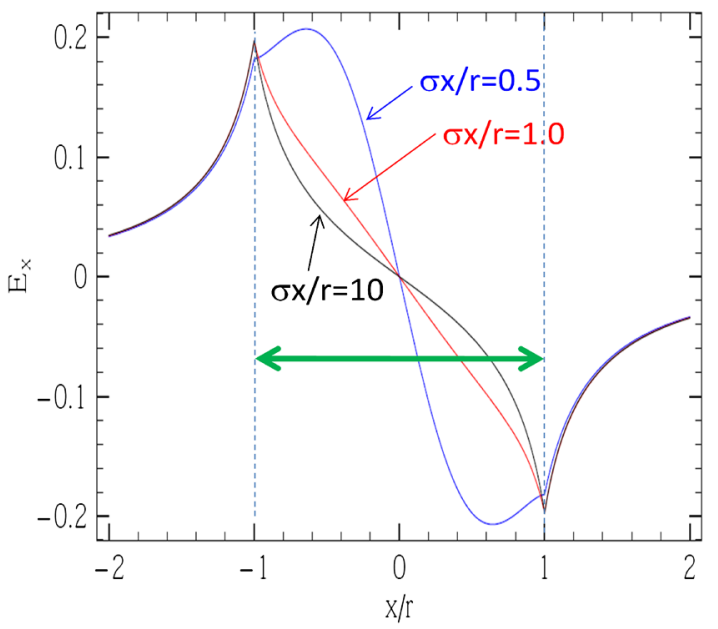

FIG. 3. Transverse space charge force for different transverse laser profiles on the cathode (by varying $\sigma_{x} / r$ ). The black line corresponds to a uniform spatial profile, the red and blue represent different truncated-Gaussian spatial profiles. The result in the red one is the most linear.

transverse iris instead of a round one, provides a qualitative understanding of the simulation results.

\section{Simulated laser transmission through the iris}

As described above, a uniform laser spatial profile requires a laser beam much larger than the iris diameter itself. In contrast, a truncated-Gaussian laser spatial profile needs a laser size comparable to or smaller than the iris, which significantly improves laser beam transmission. The laser transmission through the iris for different spatial distribution is analytically estimated, as shown in Fig. 4. All spatial distributions discussed above are treated as

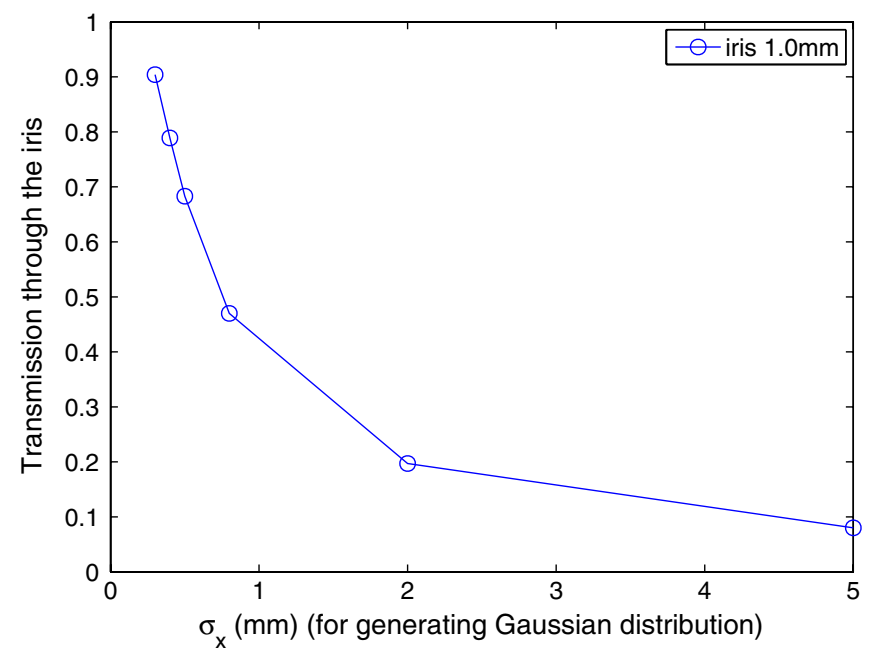

FIG. 4. Calculated laser transmission through the iris for different laser beams: the pure uniforms $\left(\sigma_{x}=5.0\right.$ and $\left.2.0 \mathrm{~mm}\right)$, nearly uniform $\left(\sigma_{x}=0.8 \mathrm{~mm}\right)$, and different truncatedGaussian spatial beams $\left(\sigma_{x}=0.5,0.4\right.$, and $\left.0.3 \mathrm{~mm}\right)$. 

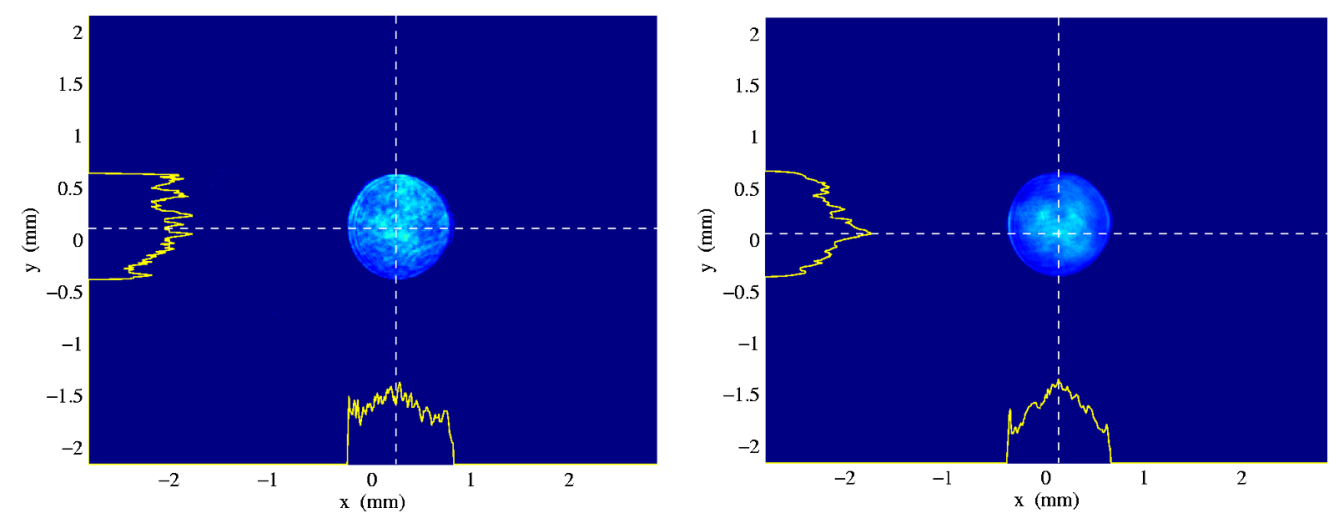

FIG. 5. Regular nearly uniform laser spatial profile (left) and truncated-Gaussian spatial profile at the cathode (right). Edge to edge laser size for both cases is $1 \mathrm{~mm}$.

Gaussian beams with different standard deviations but truncated by the same iris. The transmitted laser beam energy through the iris is the integral of the intensity of the Gaussian distribution within the iris range from -0.5 to 0.5 for a $1.0 \mathrm{~mm}$ iris. The laser transmission for the truncated Gaussian $\left(\sigma_{x}=0.4 \mathrm{~mm}\right)$ is about 1.8 times larger compared to the nearly uniform spatial profile ( $\sigma_{x}=$ $0.8 \mathrm{~mm}$ ). The result could be impacted by transverse laser jittering but a transverse feedback system for the LCLS driver laser can always keep the laser beam on the center at the iris.

\section{MEASUREMENTS}

Beam measurements were performed at the LCLS injector to compare the emittance and laser transmission using regular nearly uniform laser spatial profiles with truncated-Gaussian laser spatial beam at the cathode.
Figure 5 shows the LCLS injector drive laser spatial profiles, nearly uniform (left) and a truncated Gaussian (right). The measured laser transmission through the $1.0 \mathrm{~mm}$ iris for generating the regular nearly uniform spatial beam at the cathode was about half that for the truncated-Gaussian laser beam. The observations agree very well with the calculations shown in Fig. 4. The required laser energy from the LCLS laser amplifier for the truncated-Gaussian profile was about half that of the regular nearly uniform profile, which significantly loosens the requirements of copper cathode quantum efficiency and laser system pulse energy.

The emittance-compensating solenoid and two small quadrupoles located inside the solenoid [14] were optimized for the beam emittance generated by the laser profiles shown in Fig. 5. The projected emittance with $150 \mathrm{pC}$ charge was measured using OTR2 [14] at $135 \mathrm{MeV}$ of electron beam energy, as shown in Fig. 6. Note that 5\%
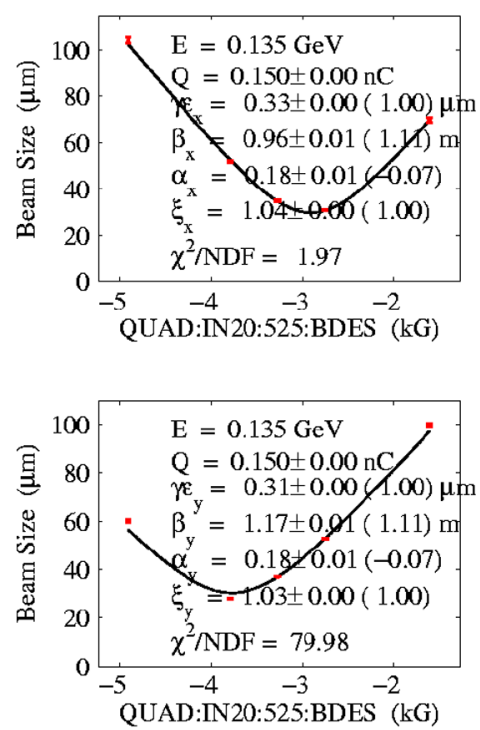
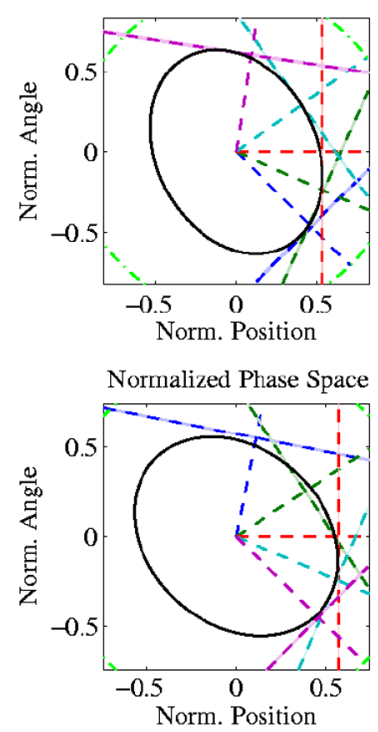
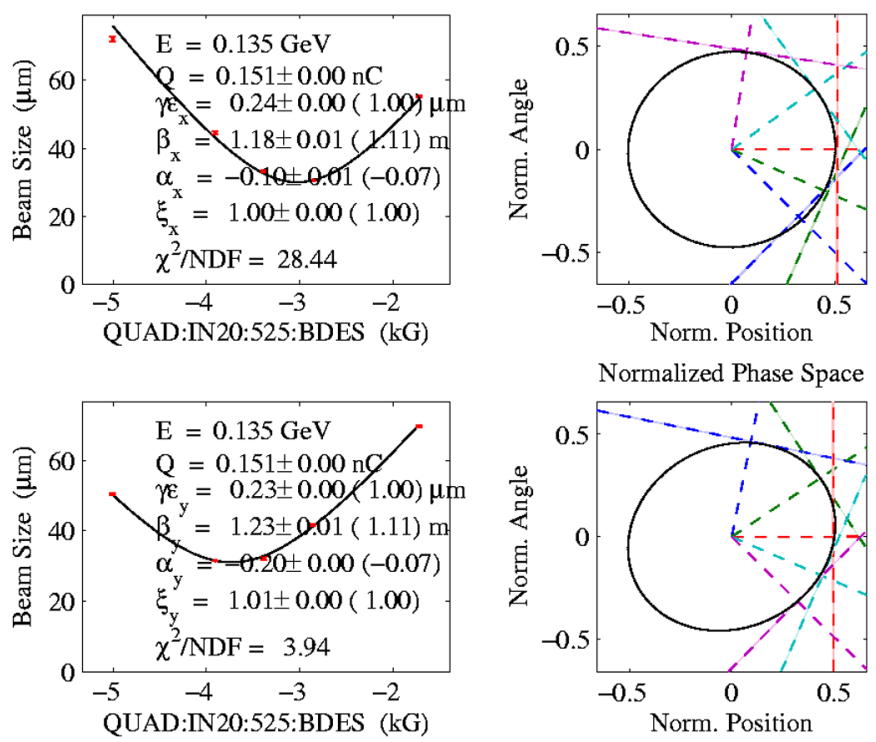

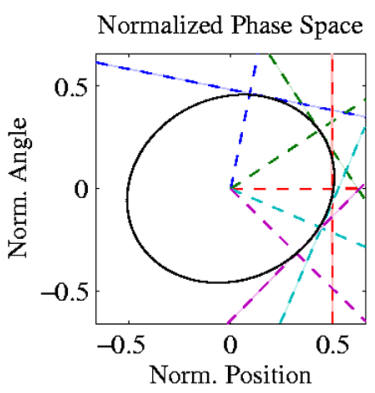

FIG. 6. Measured projected emittance at the LCLS injector with $150 \mathrm{pC}$ : regular nearly uniform (left) and truncated-Gaussian (right) laser spatial distributions at the photocathode. Note that $5 \%$ of particles in the tails are excluded in the beam size measurements. 


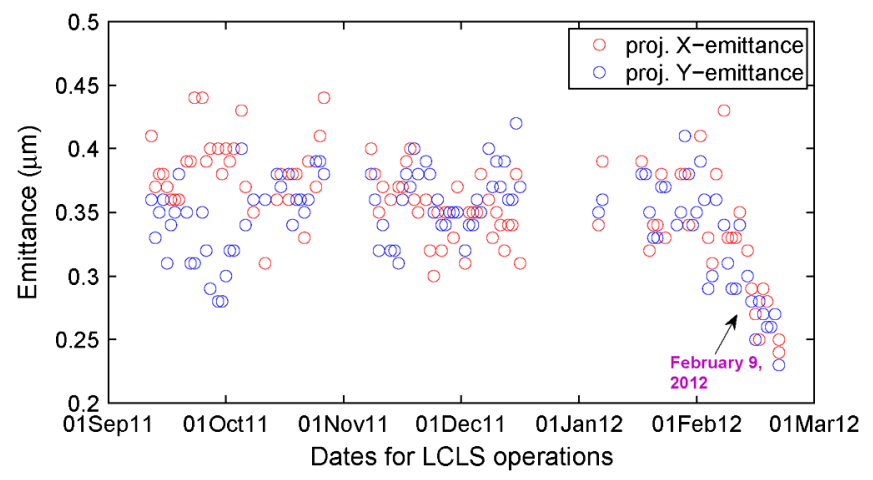

FIG. 7. The LCLS injector emittance $(150 \mathrm{pC})$ evolution during regular user operations for six months. Emittance of nearly uniform laser spatial (before February 9) and truncated-Gaussian profiles (after February 9).

of the particles in the tails are not considered in the beam size measurements. It is shown that the projected emittance is about $0.23-0.24 \mu \mathrm{m}$ (right) with the truncated-Gaussian laser spatial distribution, compared to about $0.32 \mu \mathrm{m}$ (left) using regular nearly uniform distribution. Figure 7 shows the LCLS injector emittance evolution during LCLS routine user operations with $150 \mathrm{pC}$. Before February 9, 2012, the emittance values ranged between 0.3 and $0.4 \mu \mathrm{m}$ for $150 \mathrm{pC}$ with nearly uniform laser beam profile while after February 9, 2012 the values are between 0.2 and $0.3 \mu \mathrm{m}$ for the same charge but with truncated-Gaussian laser profile. Since February 9, 2012, the truncated-Gaussian laser spatial profile has been used for the routine operations and the corresponding FEL power is at least the same as before February 9, 2012.

\section{SUMMARY}

Our simulations, theoretical analysis, and observations at the LCLS injector indicate that a truncated-Gaussian laser spatial profile at the cathode creates a lower emittance beam compared to the nearly uniform spatial profile used previously, for a given 3 ps smooth Gaussian temporal laser. This was explained using a simple analytical model that shows the transverse space charge force is more linear for the truncated-Gaussian profile. This results in an emittance reduction of $\sim 25 \%$ at the LCLS injector. Laser transmission through the iris for generating the truncated-Gaussian laser at the cathode is about twice compared to the nearly uniform one, which significantly loosens the requirements for cathode and drive laser system. Since February 9, 2012, the drive laser with the truncated-Gaussian spatial distribution at the cathode has been used for the LCLS routine user operations and the corresponding free electron laser (FEL) power is at least the same as the one using a nearly uniform spatial profile.

\section{ACKNOWLEDGMENTS}

We would like to thank LCLS physicists and the operation team for the contributions and strong support. The work is supported by DOE under Contract No. DE-AC0276 SF00515.

[1] P. Emma et al., Nature Photon. 4, 641 (2010).

[2] H. Braun, Future Light Source Workshop 2010 [http:// www-conf.slac.stanford.edu/icfa2010/].

[3] B. Carlsten, MaRIE X-ray FEL, Future Light Source Workshop 2012 [http://www.jlab.org/conferences/ FLS2012/].

[4] B. Carlsten, Nucl. Instrum. Methods Phys. Res., Sect. A 285, 313 (1989).

[5] X. Qui, K. Batchelor, I. Ben-Zvi, and X. J. Wang, Phys. Rev. Lett. 76, 3723 (1996).

[6] X. Wang, X. Qiu, and I. Ben-Zvi, Phys. Rev. E 54, R3121 (1996).

[7] F. Zhou, I. Ben-Zvi, M. Babzien, X. Y. Chang, A. Doyuran, R. Malone, X. J. Wang, and V. Yakimenko, Phys. Rev. ST Accel. Beams 5, 094203 (2002).

[8] D. Dowell, P. Bolton, J. Clendenin, P. Emma, S. Gierman, W. Graves, C. Limborg, B. Murphy, J. Schmerge, Nucl. Instrum. Methods Phys. Res., Sect. A 507, 327 (2003).

[9] D. Dowell and J. Schmerge, Phys. Rev. ST Accel. Beams 12, 074201 (2009).

[10] D. Dowell, I. Bazarov, B. Dunham, K. Harkay, C. Hernandez-Garcia, R. Legg, H. Padmore, T. Rao, J. Smedley, and W. Wan, Nucl. Instrum. Methods Phys. Res., Sect. A 622, 685 (2010).

[11] F. Stephan et al., Phys. Rev. ST Accel. Beams 13, 020704 (2010).

[12] LCLS conceptual design report, SLAC Report No. SLACR-593, 2002.

[13] A VUV FEL at the TESLA test facility at DESY: conceptual design report, DESY Report No. TESLAFEL 95-03, 1995.

[14] R. Akre, D. Dowell, P. Emma, J. Frisch, S. Gilevich, G. Hays, Ph. Hering, R. Iverson, C. Limborg, H. Loos, A. Miahnahri, J. Schmerge, J. Turner, J. Welch, W. White, and J. Wu, Phys. Rev. ST Accel. Beams 11, 030703 (2008).

[15] P. Emma (private communication).

[16] F. Zhou, A. Brachmann, F.-J. Decker, P. Emma, R. Iverson, and J. Turner, 33rd International Free Electron Laser conference, Shanghai, 2011 [http://accelconf.web .cern.ch/AccelConf/FEL2011/papers/wepa06.pdf].

[17] J. Qiang, IMPACTT code manual, Report No. LBNL-62326, 2007.

[18] Y. Ding, A. Brachmann, F.-J. Decker, D. Dowell, P. Emma, J. Frisch, S. Gilevich, G. Hays, Ph. Hering, Z. Huang, R. Iverson, H. Loos, A. Minahnahri, H.-D. Nuhn, D. Ratner, J. Turner, J. Welch, W. White, and J. Wu, Phys. Rev. Lett. 102, 254801 (2009).

[19] K. J. Kim, Nucl. Instrum. Methods Phys. Res., Sect. A 275, 201 (1989). 